INRA Prod. Anim., 2010, 23 (2), 155-166

\title{
Facteurs physiologiques et environnementaux influençant la production et la qualité de l'œuf
}

\author{
A. TRAVEL ${ }^{1}$, Y. NYS 2 , E. LOPES \\ ${ }^{1}$ ITAVI, Recherches Avicoles, F-37380 Nouzilly, France \\ 2 INRA, UR83 Recherches Avicoles, F-37380 Nouzilly, France \\ Courriel :yves.nys@tours.inra.fr
}

Le sélectionneur et l'éleveur doivent produire un œuf d'une qualité homogène et stable. Mais comment s'affranchir des variations induites par la physiologie de la poule ? De nombreuses stratégies, génétique ou nutritionnelle, ont été mises en œuvre pour limiter l'augmentation du poids d'œuf ou pour contrôler la dégradation de la qualité de l'œuf associées à l'âge de la poule. L'environnement, notamment la lumière et la température, affecte également la physiologie de la poule et ses performances. Ces facteurs sont contrôlés pour optimiser la qualité de l'œuf.

L'œuf est un produit élaboré, de structure et de composition parfaitement connues. Son élaboration est soumise à un contrôle physiologique précis des organes reproducteurs de la poule, selon une séquence temporelle et spatiale définie (encadré 1) (Sauveur 1988, Nys 2010). Une poule mature d'une lignée commerciale produit près d'un œuf par jour. L'œuf ainsi formé contient l'ensemble des nutriments indispensables au développement d'un embryon dans le milieu extérieur. Ses qualités nutritionnelles en font un produit de grande consommation, prisé par les consommateurs qui exigent une qualité irréprochable. Celle-ci dépend de facteurs directement liés à l'animal (génétique, âge de la poule), de son alimentation et des conditions de son environnement (système d'élevage, lumière et température). Dans cet article, nous nous intéresserons aux facteurs physiologiques (âge de la poule, mue) et environnementaux (programmes lumineux, température) qui influent sur la physiologie de la poule et par conséquent sur la qualité des œufs. De plus, nous évaluerons l'impact du système d'élevage. Les critères qualitatifs retenus dans cet article sont ceux souhaités par les consommateurs d'œufs de qualité A : la qualité extérieure (propreté, couleur, homogénéité externe, intégrité et solidité de la coquille), le calibre de l'œuf et sa qualité interne (couleur du jaune, hauteur du blanc épais, absence d'inclusions, composition de l'œuf et caractéristiques fonctionnelles). Beaucoup de références décrivant l'influence de la physiologie de la poule ou de son environnement sur la qualité de l'œuf ont plus de 20 ans donc décrivent des expérimentations conduites sur des génotypes bien différents de ceux d'aujourd'hui.

\section{1 / Facteurs liés à la poule}

\section{1 / Âge de la poule}

a) Poids d'œuf et part des compartiments

Le poids de l'œuf varie de 50 à $70 \mathrm{~g}$ (extrême de 45 à $75 \mathrm{~g}$ ) principalement avec l'âge et secondairement avec le croisement commercial de la poule. Le poids de l'œuf augmente considérablement au cours de l'année de production mais cette évolution ainsi que le poids moyen de l'œuf dépend de la lignée de poule, notamment en liaison avec son poids corporel. Cette augmentation a été fortement limitée par la sélection pour les lignées commerciales actuelles. Le poids de l'œuf brun variait de 53 à $67 \mathrm{~g}$ entre 30 semaines et la fin de production en 1981, contre une variation de 60 à $65,5 \mathrm{~g}$ pour la même période en 2007
(Nys et al 2008). En effet, le poids des œufs d'une jeune poule atteint $60 \mathrm{~g}$ à 26 semaines puis tend à se stabiliser à $65 \mathrm{~g}$ à partir de 50 semaines. Il s'élève à environ $68 \mathrm{~g}$ vers 80 semaines d'âge (Beaumont et al 2010).

L'augmentation du poids de l'œuf au cours d'un cycle de ponte, liée au vieillissement de la poule, s'accompagne d'une augmentation de la part relative de jaune et d'une diminution de la part de la coquille (tableau 1, Ternes et al 1994). Globalement, les proportions de jaune, de blanc et de coquille varient respectivement de 25 à $33 \%$, de 57 à $65 \%$ et de 8,5 à $10,5 \%$.

Le poids de l'œuf est un aspect qualitatif de grande importance économique : les œufs de catégorie A sont vendus par classe de poids (ou calibre). L'importance de ce critère de vente explique l'intérêt des producteurs à allonger la période d'élevage en vue de produire des œufs de «gros calibre». Une alternative pour obtenir un poids d'œuf élevé, très utilisée aux USA

Tableau 1. Evolution du poids de l'œuf et des différents compartiments de l'œuf au cours de l'année de production (valeurs moyennes arrondies de 900 œufs par groupe) (Ternes et al 1994).

\begin{tabular}{|l|c|c|c|c|c|c|}
\hline \multirow{2}{*}{ Âge des poules } & \multicolumn{2}{|c|}{$\mathbf{3 4 / 3 5}$} & \multicolumn{2}{c|}{$\mathbf{5 0 / 5 1}$} & \multicolumn{2}{c|}{$\mathbf{7 0 / 7 1}$} \\
\cline { 2 - 7 } (semaines) & $\mathbf{( g )}$ & $\mathbf{( \% )}$ & $\mathbf{( g )}$ & $\mathbf{( \% )}$ & $\mathbf{( g )}$ & $\mathbf{( \% )}$ \\
\hline CEuf & 61 & & 66 & & 68 & \\
Jaune & 16 & 26 & 19 & 29 & 20 & 29 \\
Coquille & 6,1 & 10 & 6,6 & 10 & 6,7 & 9,85 \\
Blanc & 39 & 63 & 41 & 61 & 41 & 61 \\
\hline Rapport blanc/jaune & \multicolumn{2}{|c|}{2,4} & \multicolumn{2}{|c|}{2,2} & \multicolumn{2}{|c|}{2,1} \\
\hline
\end{tabular}


Encadré 1. Formation de l'œuf

L'œuf est formé en deux étapes principales. Tout d'abord, la majeure partie du jaune résulte de l'accumulation en une dizaine de jours, dans les follicules de l'ovaire, de lipoprotéines synthétisées par le foie. Le plus gros follicule est ovulé dans l'oviducte sous l'influence d'hormones hypophysaires qui interagissent avec les stéroïdes ovariens pour synchroniser cette ovulation du jaune peu après l'expulsion de l'œuf précédent (oviposition). Ensuite, l'œuf en formation acquiert en 24 h (figure 1) ses différents compartiments dans les différents segments de l'oviducte: le blanc, les membranes coquillières puis la coquille sont déposés successivement par le magnum, l'isthme et l'utérus.

(au contraire de la France) est l'induction d'une mue pour mettre en place un deuxième cycle de production.

\section{b) Qualité extérieure de l'œuf}

- La forme et l'aspect des œufs

L'indice de forme de la coquille (L/1 de l'œuf) augmente avec l'âge des poules pondeuses (Romanoff et Romanoff 1949). En début de production, les œufs ont plutôt une forme ronde qui tend progressivement à s'allonger au cours de l'année de ponte. Cette modification de la forme de l'œuf résulterait d'un affaiblissement de la tonicité musculaire de la glande coquillière chez les poules âgées.

En début de période de ponte, apparaissent parfois des œufs à double jaune, anormalement grands et de forme allongée résultant d'une double ovulation synchrone. La fréquence de ces «œufs doubles» diminue progressivement au cours du cycle de ponte. La proportion de petits œufs peut également être importante en début du cycle de production si la maturité sexuelle a été trop précoce, du fait des programmes lumi- neux et alimentaires mis en place en période d'élevage. Occasionnellement, en début de ponte, on remarque la présence de très petits œufs sphériques ne contenant que du jaune d'œuf.

La couleur de la coquille, notamment pour les œufs bruns, s'éclaircit avec le vieillissement des poules (Mills et al 1991).

\section{- Défauts de présentation}

- Des défauts de présentation de coquilles peuvent être observés chez de jeunes poules en début de production malgré les efforts en sélection. Ils sont décrits dans des revues de différents auteurs (Romanoff et Romanoff 1949, Sauveur 1988, Mertens et al 2010) ;

- Les œufs présentent un disque aplati et non coloré en surface de coquille, à proximité d'un pôle ;

- Les œufs ont une coquille molle ou même absente. Ces deux défauts résultent du télescopage de deux œufs dans l'utérus du fait de deux ovulations en moins de $24 \mathrm{~h}$, la deuxième se produisant bien avant l'expulsion du premier œuf en formation dans l'oviducte ;

- La présence d'un anneau au niveau équatorial résulte d'une réparation in utero d'une fracture précoce de la coquille encore molle, due probablement à un stress des poules induisant des contractions de l'utérus à ce stade précoce de formation de la coquille en début de nuit. Ce défaut n'est pas directement lié à l'âge des poules ;

- Les troupeaux âgés, quant à eux, tendent à produire des œufs avec des dépôts sablonneux, des coquilles crayeuses ou présentant des aspérités de quelques millimètres. Cette dernière particularité résulte d'un défaut interne au niveau des membranes coquillières (inclusions déformant la membrane coquillière).

\section{- Solidité de la coquille}

Le taux d'œufs cassés et fêlés est faible en début de production puis augmente au cours d'un cycle de ponte, pour atteindre à la fin d'une année de production des valeurs autour de 12 voire autour de $20 \%$ dans certains élevages, selon les conditions de management, d'alimentation et d'environnement (Sauveur 1988, Nys 2010). Ce taux de déclassement est relativement stable malgré les efforts de sélection car, parallèlement, la sensibilité des techniques de détection des œufs fêlés a progressé. Ces défauts de non intégrité de la coquille augmentent le risque de toxi-infection alimentaire car ils favorisent la pénétration de bactéries pathogènes dans l'œuf.

Figure 1. Représentation de l'oviducte de poule et de la séquence spatiale et temporelle de la formation de l'œuf (Nys 2010 d'après Romanoff et Romanoff 1949).

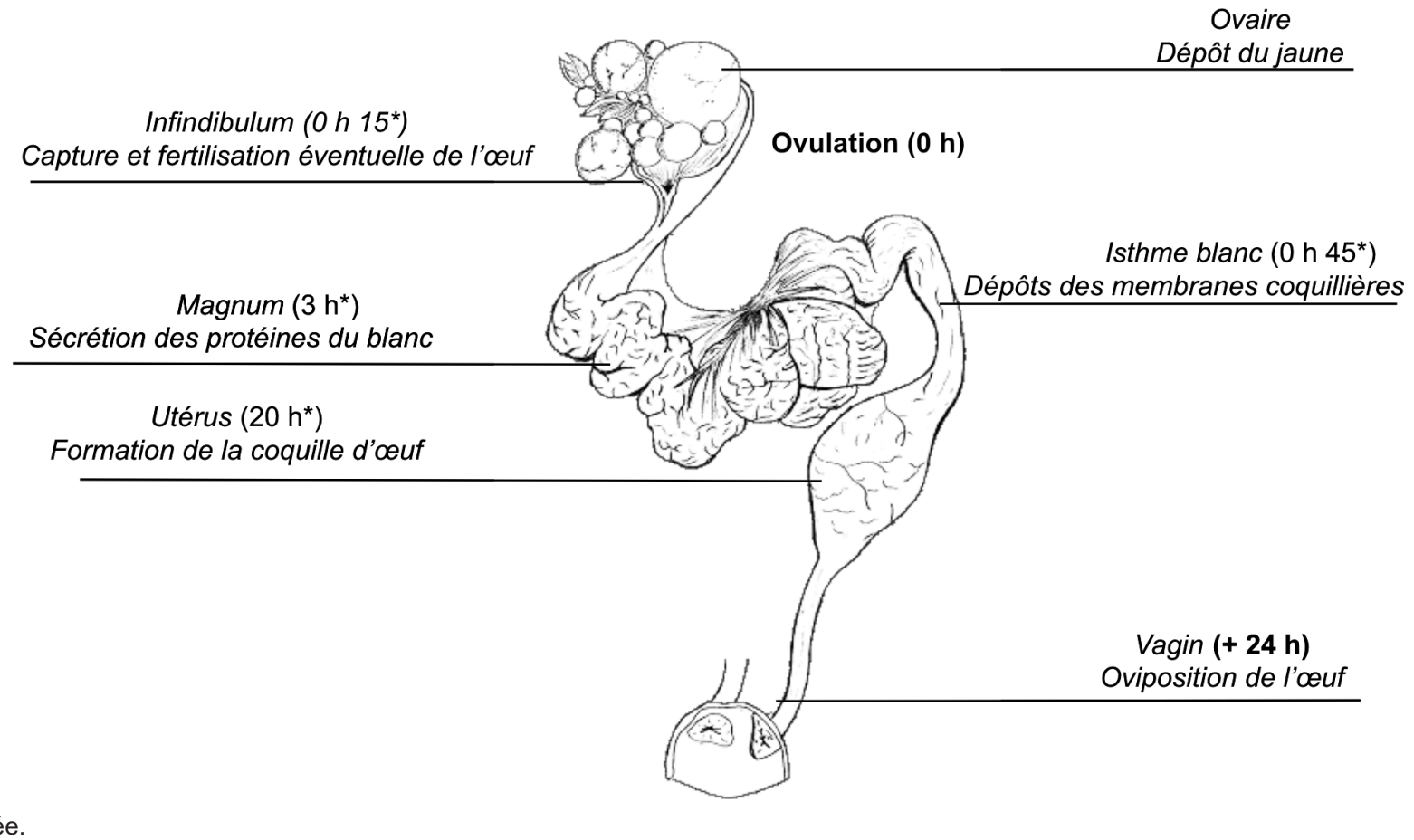


Figure 2. Influence de l'âge des poules sur la hauteur du blanc d'œuf épais (exprimée en unité Haugh) (ADAS 1989).

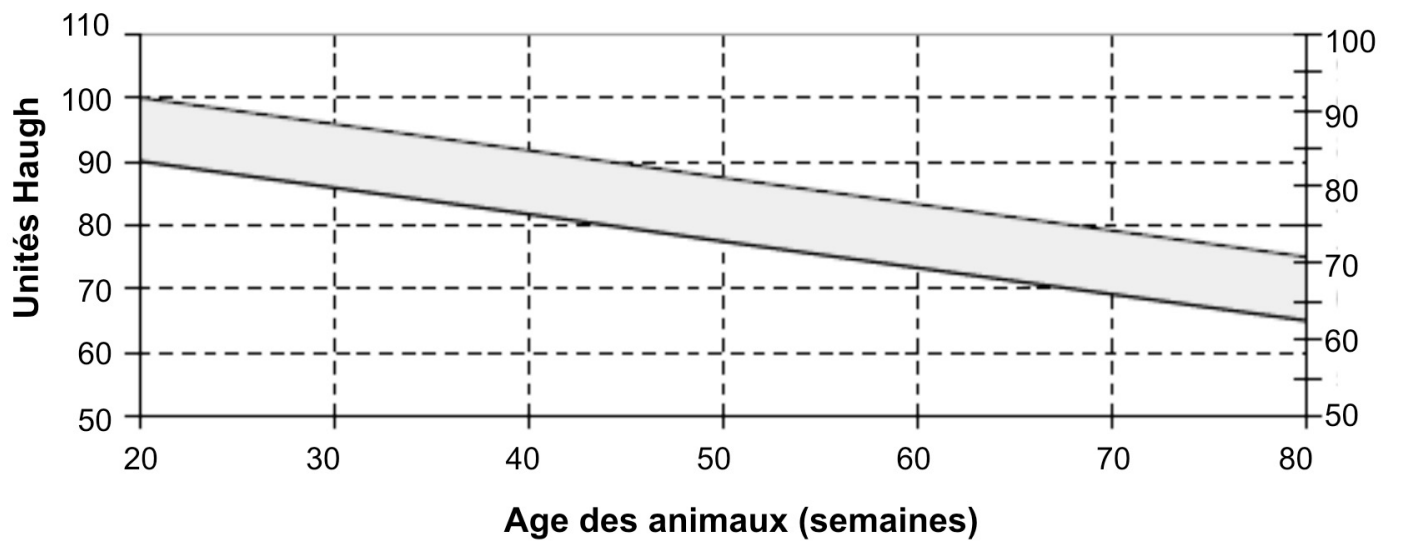

Lorsque le poids de l'œuf augmente avec l'âge de la poule, le poids de coquille s'accroît moins vite que celui de l'œuf, ce qui se traduit par une baisse du pourcentage de coquille ou de la gravité de l'œuf ${ }^{1}$ relativement rapide en début de ponte (avant 36 semaines) et qui se ralentit par la suite (Sauveur 1988, Curtis et al 2005). Pour un poids d'œuf donné, par exemple de $62 \mathrm{~g}$, le poids de coquille est de $5,80 \mathrm{~g}(9,3 \%)$ à 37 semaines mais seulement de $5,55 \mathrm{~g}$ $(8,9 \%)$ à 70 semaines d'âge. A un âge donné, lorsque le poids de l'œuf augmente, la part de coquille diminue également : à 37 semaines, la coquille augmente de 5,3 à $6,1 \mathrm{~g}$ si le poids de l'œuf varie de 56 à $66 \mathrm{~g}$. Il est à noter cependant que la durée de dépôt de la coquille ne varie pas avec l'âge de la poule, alors que l'intervalle entre deux ovipositions s'accroît notablement au fur et à mesure que l'intensité de ponte diminue (Nys 1986).

Il existe une excellente relation entre le poids de coquille et la résistance à la rupture de la coquille d'un œuf entier de poule soumis à un test de compression statique (Mertens et al 2010). Néanmoins, la solidité de la coquille dépend non seulement de son épaisseur mais également de son organisation ultrastructurale et cristallographique (Nys et al 1999). Chez les poules âgées, il existe des modifications de l'organisation de la microstructure au niveau de la couche mamillaire (profondeur de la jonction entre les cônes adjacents, par exemple) qui influencent directement la liaison entre la coquille calcifiée et les membranes sous-jacentes fragilisant la coquille (Solomon 1991). De plus, les coquilles d'œufs de poules âgées, moins solides, présentent davantage de variabilité de leurs propriétés texturales (taille et orientation des cristaux) et se caractérisent par une dominance plus marquée d'un degré d'orientation cristalline favorisant l'apparition de plans de clivage (Rodriguez-Navaro et al 2002). Enfin, un œuf de poule jeune est plus rond qu'un œuf en fin de cycle de production et se révèle donc plus résistant à une pression statique.

\section{c) Composition de l'œuf \\ - Part des compartiments}

Les variations du poids de l'œuf au cours de l'année de ponte de la poule ainsi que de la part relative des différents compartiments affecte le rapport blanc/jaune (tableau 1). Cette proportion constitue la source la plus importante de variation de la composition de l'œuf, du fait de la composition très différente de ces deux compartiments. Cependant, la sélection génétique a réduit la variabilité de la part des compartiments de l'œuf en fonction de l'âge, comparé à des souches plus anciennes (Curtis et al 2005).

\section{- Composition chimique}

Un effet décrit antérieurement de l'âge de la poule est la réduction de la matière sèche du blanc de 0,5 à 1,5 points (d'environ 13 à $12 \%$ de MS en fin de ponte) de sorte que l'augmentation du poids de blanc avec l'âge ne se traduit que par une faible variation de la matière sèche totale. En revanche, celle du jaune reste relativement stable. Quant à la matière sèche du mélange, elle augmente pendant les deux premiers mois puis se stabilise à 25\% (Sauveur 1988). Ces données sont cependant anciennes et mériteraient d'être réévaluées car, actuellement, les références industrielles de matière sèche du mélange sont d'environ 22,5 à $23 \%$ pour le mélange et voisine de 10,5 à $11 \%$ pour le blanc (SNIPO 2008).

Les données obtenues sur l'évolution de la composition de constituants de l'œuf en fonction de l'âge de la poule sont, pour la plupart, peu reproductibles. $\mathrm{Si}$ les teneurs en protéines et lipides totaux des compartiments de l'œuf sont reconnues comme très peu variables, des résultats totalement contradictoires ont été rapportés concernant les contenus de l'œuf en cholestérol et en acides gras essentiels, souvent par manque de prise en compte de la part de jaune elle-même. En revanche, si l'augmentation du poids de jaune avec l'âge est prise en compte, le contenu total en lipides d'un œuf augmente d'environ un tiers, passant de 6 à $9 \mathrm{~g}$, entre un «petit» et un «gros» œuf entre un début et une fin de production.

Lorsque l'œuf est enrichi en acide linolénique (C18:3, n-3) par l'alimentation de la poule, le taux de transfert serait plus élevé chez des poules âgées de 58 semaines par rapport à des poules de 36 semaines d'âge (Scheideler et al 1998).

d) Qualité physique et caractéristiques fonctionnelles

Parallèlement à l'augmentation du poids de l'œuf au cours du cycle de ponte, la part de blanc épais s'accroît au détriment du blanc liquide interne. La qualité moyenne de l'albumen (unité Haugh qui reflète la part de blanc épais) régresse avec le vieillissement du troupeau (Sauveur 1988, Curtis et al 2005) (figure 2).

La structure de la membrane périvitelline change également avec l'augmentation de la taille du follicule. En fin de ponte, sur de gros œufs, la capacité de cette membrane à garder son intégrité peut alors être réduite induisant une détérioration plus rapide du jaune d'œuf. Par conséquent, le jaune d'œuf se casse facilement lors de sa séparation du blanc. Curtis et al (2005) observent une diminution de la résistance de la

\footnotetext{
${ }^{1}$ La densité de la coquille étant deux fois plus élevée que celle du contenu, la densité de l'œuf par flottaison varie avec la quantité de coquille.
} 
Figure 3. Taux de ponte (\% d'œufs commercialisés donc après retrait des œufs déclassés) enregistrés sur 25 millions de pondeuses en 1998 aux USA au cours de trois cycles successifs (Bell 2003).

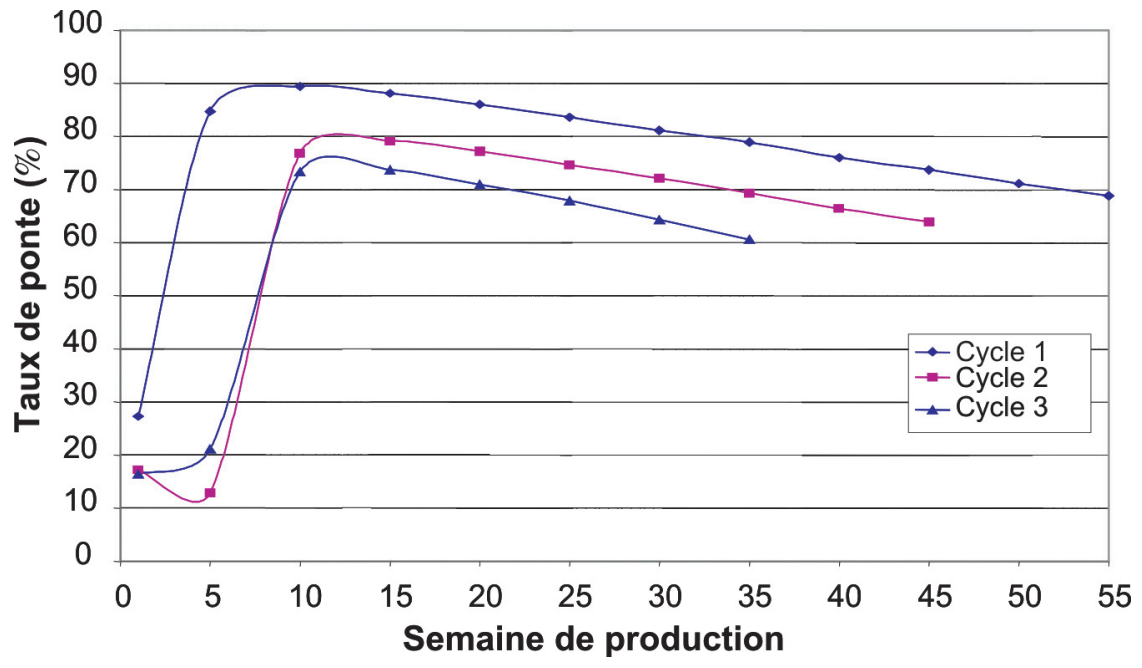

membrane vitelline de 2,33 à $1,92 \mathrm{~g}$ entre le début et la fin de ponte.

Une étude conduite par Kerth et al (2005) a montré que l'âge des poules modifie le pouvoir émulsifiant du jaune. La résistance d'une émulsion de mayonnaise augmente au cours des 5 premiers mois de production de 386 à $512 \mathrm{~g}$, puis se stabilise au cours des 7 derniers mois (438 à $508 \mathrm{~g}$ ). Ces auteurs ont également observé des variations de volume des gâteaux de Savoie préparés avec des œufs de début de ponte $(339 \mathrm{~mL})$ et des œufs de fin de ponte $(270 \mathrm{~mL})$. Les œufs apparaissent donc comme des produits variables qui subissent une perte de qualité notamment de l'albumen et de la coquille, avec l'âge du troupeau.

\section{2 / La mue}

Décrit avec précision par Sauveur (1988), la mue est un phénomène physiologique naturel de renouvellement du plumage chez les oiseaux. Cette mue s'accompagne, chez les poules en production, d'un arrêt de ponte. Elle peut être naturelle (effet saison) après la période de reproduction et la période d'incubation qui précède la migration pour les oiseaux sauvages. Elle est déclenchée en fin d'année de production en élevage de poules pondeuses afin de restaurer les performances du troupeau et la qualité des œufs, dégradée en fin de cycle de ponte. La décision de procéder à une mue ou de renouveler le troupeau est économique et prend en compte les performances du troupeau ainsi que les cours du prix de l'aliment, des oufs et des poulettes (Bell 2003). Cette technique est appliquée en général entre 60 et 80 semaines d'âge selon les performances, et permet de prolonger le troupeau jusqu'à 110
6 semaines, notamment la distribution à volonté d'un aliment pauvre en sodium ou calcium, ou enrichi en minéraux (zinc, aluminium, potassium), ou bas en énergie et/ou riche en fibres (insolubles) via l'incorporation de coproduits de luzerne (Landers et al 2005), de blé, de graines de coton, de jojoba ou de son de riz, par exemple (Mansoori et al 2007). Ces stratégies alimentaires induisent systématiquement une réduction plus ou moins marquée de la consommation. Ces approches synchronisent la mue au sein d'un troupeau. La durée de la mue et de l'arrêt de la ponte, la mortalité et l'intensité de la reprise dépendent du programme de mue choisi.

\section{a) Production et poids des $œ u f s$}

La production d'œufs pendant la mue chute à $5 \%$ pendant environ 3 semaines. Après la mue, la production d'œufs est améliorée par rapport à la période précédant la mue et la quantité d'œufs pondus par poule est augmentée. Le second pic de ponte est atteint après 13 semaines dans le deuxième cycle (entre 75 et $85 \%$ ) et après 13 semaines dans le troisième cycle $(74,7 \%)$. La persistance de production est diminuée par rapport au $1^{\text {er }}$ cycle. Les courbes de ponte sont comparables pour le 1 er et $2^{\text {ème }}$ cycle, mais avec des évolutions plus rapides dans le 3 ème cycle de production (figure 3 ).

En cas de mue forcée après environ un an de production, le poids des $1^{\text {ers }}$ œufs pondus lors du second cycle est élevé dès le début de ponte (63-65 g environ, figure 4 ). Il est en moyenne supérieur de 4 à $5 \mathrm{~g}$ à celui obtenu en première ponte et plafonne à partir du 4ème mois de la deuxième ponte (Sauveur 1988, Ahmed et al 2005).

\section{b) Qualité interne de l'œuf}

De même que pour un $1^{\text {er }}$ cycle de ponte, les changements de proportion des compartiments de l'œuf sont accentués après une mue. La réduction du rapport blanc/jaune est davantage accentuée lors d'un $2^{\text {ème }}$ cycle de production. Les œufs de 2 ème ponte, relativement plus riches en jaune, sont donc très intéressants pour l'extraction et/ou le traitement du vitellus. La qualité de l'albumen se révèle également améliorée après une mue mais se dégrade plus rapidement au cours du deuxième cycle (Sauveur 1988).

\section{c) Solidité et forme de coquille}

De nombreuses publications démontrent une amélioration de la qualité des coquilles d'œufs après la mue et notamment, après une mue artificielle (Garlich et al 1984, Bell 2003). Garlich et al (1984), en accord avec d'autres auteurs, associent cette amélioration de 
Figure 4. Evolution du poids moyen de l'œuf lors des cycles de production 1, 2 et 3 (Bell 2003).

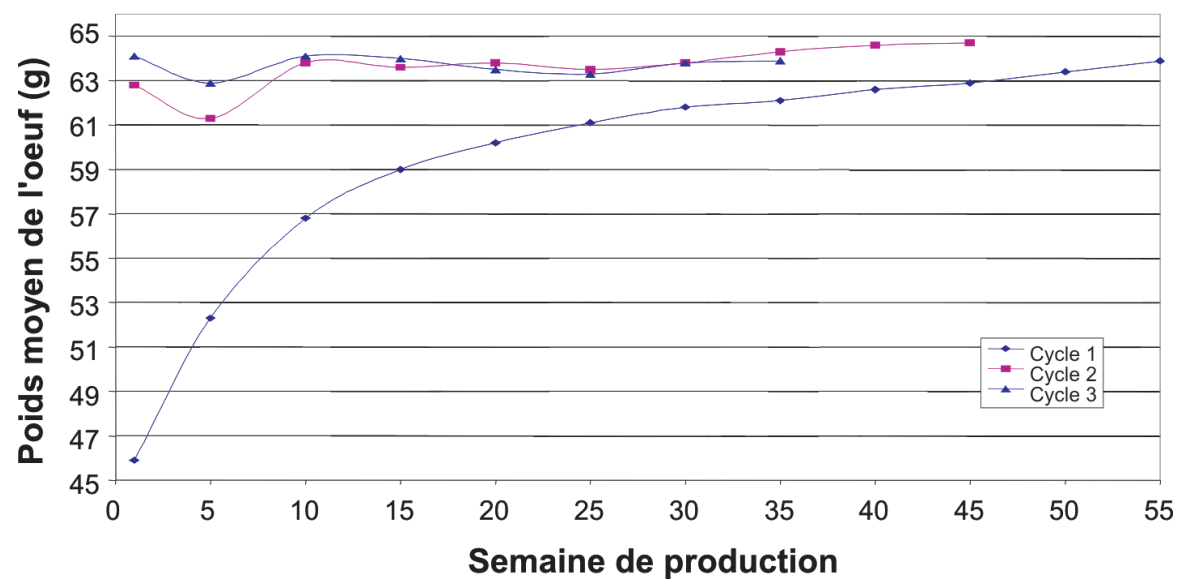

la solidité des coquilles après la mue à celle de l'épaisseur de la coquille. Selon Roberts et Brackpool (1994), la mue a des effets limités sur la microstructure de la couche mamillaire (fusion entre cônes). Par contre, Ahmed et al (2005) ont montré, en comparant un échantillon d'œufs de même taille et de même poids de coquille, que la mue améliore les propriétés mécaniques de la coquille indépendamment de son épaisseur. Cette amélioration coïncide avec une réduction de la taille des cristaux de calcite et avec un enrichissement de la matrice organique en protéines spécifiques de la coquille.

\section{2 / Les facteurs environne- mentaux}

La distinction entre les effets directs de l'environnement sur la qualité de l'œuf via des modifications de la physiologie de la poule et ceux induits indirectement à travers des modifications pour la consommation alimentaire n'est pas toujours aisée. Dans ce chapitre, nous explorerons l'impact de la température, des programmes lumineux et du système d'élevage sur la qualité des œufs.

\section{1 / Impact de la température}

En élevage, la température requise pour une production optimale des poules pondeuses se situe aux alentours de $22-24^{\circ} \mathrm{C}$ (Mardsen et Morris 1987) mais les poules possèdent une bonne tolérance à des températures inférieures. Cependant, un stress thermique brutal, cyclique ou constant à une température supérieure à $29^{\circ} \mathrm{C}$ modifie le métabolisme de l'animal (encadré 2), réduit son ingéré alimentaire et entraîne des effets néfastes sur les performances des volailles et plus particulièrement sur la production et la qualité des œufs (Marsden et Morris 1987, Sauveur formation de la coquille. des tissus périphériques (Yahav 2009). voie d'élimination de la chaleur du fait de l'absence, chez l'oiseau, de glandes sudoripares.

\section{a) Consommation d'aliment}

Le besoin d'entretien de la poule, qui se traduit directement par une modification de l'ingéré alimentaire, diminue de $4 \%$ par degré $\left({ }^{\circ} \mathrm{C}\right)$ au-dessus de la zone de neutralité thermique (Sauveur 1988). De plus, la poule réduit préférentiellement sa thermogenèse en cas de hautes températures. Aux alentours de $30^{\circ} \mathrm{C}$, la consommation d'aliment, ou l'ingéré d'énergie métabolisable, et par conséquent le poids corporel diminuent notablement pour les poules exposées de manière constante à de fortes températures. Cette relation entre température et ingéré d'énergie métabolisable se révèle curvilinéaire (Mardsen et Morris 1987). Selon ces auteurs, prenant en compte diverses références, l'ingéré d'aliment des pondeuses diminuerait de 1 à $1,5 \%$ par degré entre 20 et $30^{\circ} \mathrm{C}$ et de $5 \%$ par degré entre 32 et $38^{\circ} \mathrm{C}$. Cette réduction apparaît donc 4 fois supérieure à forte température par rapport à celle prévalent dans la zone de neutralité thermique (Picard et al 1993). La consommation

\section{Encadré 2. Altération physiologique induite par la chaleur chez la poule}

La poule est un animal homéotherme, qui maintient sa température corporelle constante autour de $41^{\circ} \mathrm{C}$ pour un fonctionnement normal de ses organes vitaux. La «zone de neutralité thermique» (zone délimitée par les températures ambiantes qui ne modifient pas la température corporelle) se situe entre 10 et $20^{\circ} \mathrm{C}$. La poule pondeuse a, cependant, une tolérance assez large car sa température corporelle n'est pas affectée par un spectre large de températures ambiantes. Cette zone apparaît plus étroite en termes de productivité optimale étant donné que la poule surconsomme à des températures basse $\left(<15^{\circ} \mathrm{C}\right)$ et sous-consomme à température élevée $\left(>22-25^{\circ} \mathrm{C}\right)$. L'hygrométrie ainsi que la vitesse de l'air dans le bâtiment modifient la température ressentie par l'animal et influencent leur confort thermique, principalement en dehors de la zone de neutralité thermique.

Une poule soumise à haute température augmente, dans un premier temps, le flux sanguin vers ses tissus périphériques (réduction jusqu'à $45 \%$ de la circulation viscérale) dépourvus de plumes (barbillon, crête, pattes) afin d'éliminer sa production de chaleur par radiation et conduction (Sauveur 1988, Yahav 2009). Cette première phase se traduit par une réduction du rythme cardiaque, une vasodilation au niveau des organes périphériques, une réduction de la pression artérielle. Elle conduit à une compensation au niveau cardiaque par une augmentation des volumes sanguins. Une température plus élevée $\left(>29^{\circ} \mathrm{C}\right.$ ou même dès $27^{\circ} \mathrm{C}$ en cas d'hygrométrie élevée) accélère le rythme cardiaque et respiratoire provoquant une hyperventilation respiratoire (halètement (panting), jusqu'à 160 cycles par minute au lieu de $30)$. Celle-ci permet une élimination de chaleur par évaporation d'eau ( $6 \mathrm{kcal}$ évacuées pour $1 \mathrm{~g}$ d'eau évaporée). Ce mécanisme est d'autant plus efficace que l'hygrométrie est faible. Le halètement provoque une déshydratation importante des poules (élimination de 5 à $18 \mathrm{~g}$ d'eau/h) compensée par une consommation d'eau plus importante. Elle est également à l'origine d'une élimination excessive de $\mathrm{CO}_{2}$ induisant une réduction de sa teneur au niveau plasmatique et d'une augmentation du $\mathrm{pH}$. Cette alcalose respiratoire favorise l'élimination des ions bicarbonates par les reins et réduit leur disponibilité ainsi que celle du $\mathrm{CO}_{2}$, précurseurs indispensables à la formation des carbonates de la coquille (Mongin 1978). La baisse d'activité de l'anhydrase carbonique (Goto et al 1979) et du flux sanguin dans l'utérus (- $40 \%$, Wolfenson et al 1981) renforce l'effet négatif de cette alcalose sur la

La poule présente néanmoins une capacité limitée à éliminer la chaleur. Ainsi, elle compense les effets de la chaleur en réduisant sa thermogenèse principalement par diminution de l'extra-chaleur en lien avec l'alimentation. Celle-ci est induite par l'ingestion, la digestion et l'utilisation métabolique des nutriments. Aussi, pour limiter l'augmentation de leur température corporelle, les pondeuses diminuent leur consommation d'aliment (Picard et al 1993). Cette réduction de la thermogenèse est régulée par les hormones thyroïdiennes, notamment par une réduction de la déiodination de la thyroxine (T4) pour former la T3 au niveau 
Figure 5. Evolution du poids moyen des œufs de poules pondeuses soumises à une chaleur cyclique (de $27^{\circ} \mathrm{C}$ à $33^{\circ} \mathrm{C}$ ) (Travel et al 2009).

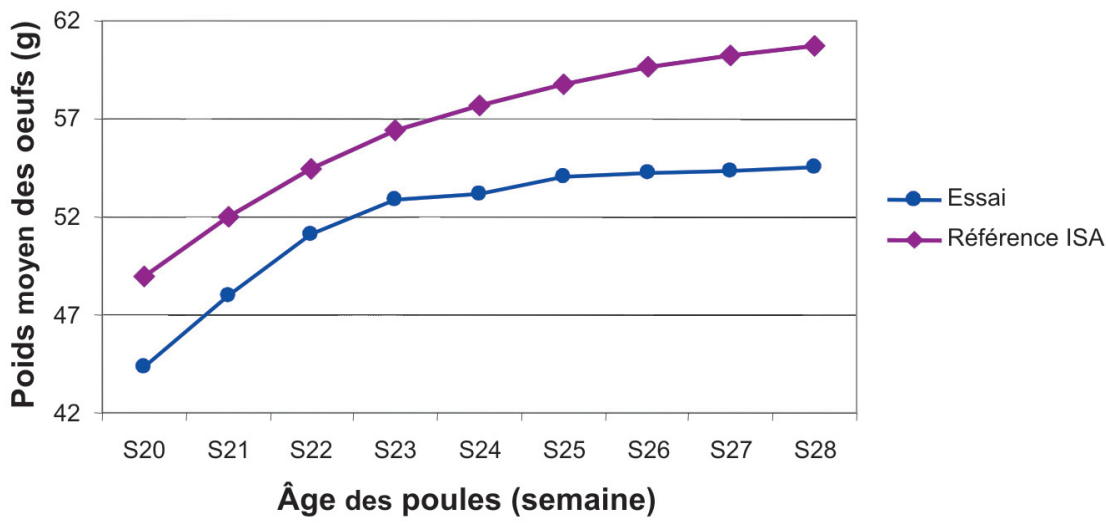

d'eau augmente au-delà de $20^{\circ} \mathrm{C}$. Elle est multipliée par deux entre 21 et $32^{\circ} \mathrm{C}$, par trois entre 21 et $37^{\circ} \mathrm{C}$ (Balnave et Bracke 2005). Notons que l'effet de la température sur la consommation d'aliment et la production d'œufs est moins marqué lorsque les fortes températures sont cycliques comparées à une chaleur constante. Une ventilation adaptée augmente l'élimination de chaleur par les animaux et, lorsqu'elle est combinée à un refroidissement par évaporation d'eau, favorise l'apparition de cycle de température et réduit l'impact négatif des fortes températures (Balnave et Bracke 2005).

\section{b) Taux de ponte et poids de l'ouf}

La production d'œufs se révèle maximale dans la zone de neutralité thermique. Elle chute d'une manière importante (de plus de 20 points) lorsque les poules sont exposées de manière constante à de fortes températures $\left(30^{\circ} \mathrm{C}\right)$ si l'aliment n'est pas modifié (Sauveur 1988, Balnave et Brake 2005). Le pic de ponte est diminué et la courbe de ponte décline plus rapidement que celle obtenue en température normale. Cependant, l'introduction d'aliment très concentré permettrait d'assurer une production normale jusqu'à $25^{\circ} \mathrm{C}$ voire $29^{\circ} \mathrm{C}$ pour des lignées d'œufs blancs. En cas de stress thermique modéré, une augmentation des niveaux de protéines et d'acides aminés essentiels permettent de compenser l'effet négatif des températures élevées (Balnave et Brake 2005). En revanche, à température très élevée, l'augmentation de la teneur énergétique dans l'aliment et du taux de protéines ne compensent pas les chutes de performances (Picard et al 1993). Selon ces mêmes auteurs, un excès de protéines peut même aggraver la situation si un acide aminé est limitant.

L'application d'une chaleur cyclique (de 27 à $33^{\circ} \mathrm{C}$ ) sur $24 \mathrm{~h}$, entre 20 et 28 semaines d'âge, affecte significativement le poids moyen des œufs, en comparaison avec les performances d'ISA Brown (figure 5). Ceci est principalement à mettre en relation avec une faible consommation alimentaire (autour de $85 \mathrm{~g}$ par jour par animal). Néanmoins, la chaleur cyclique n'a pas eu d'impact significatif sur la production d'œufs (Travel et al 2009).

Picard et al (1993) ont montré que des poules soumises à de fortes températures pendant plusieurs mois retrouvent rapidement une consommation d'aliment et des capacités de production normales lorsque la température ambiante redescend de 32 à $22^{\circ} \mathrm{C}$.

Une élévation de température d'élevage au-delà de $16^{\circ} \mathrm{C}$ se traduit par une réduction du poids d'œuf qui est curvilinéaire. La diminution varie de $0,4 \mathrm{~g} /{ }^{\circ} \mathrm{C}$ à près $\mathrm{d}^{\prime} 1 \mathrm{~g} /{ }^{\circ} \mathrm{C}$ pour des températures supérieures à $25^{\circ} \mathrm{C}$. Selon Smith et Oliver (1972), au-delà de $25^{\circ} \mathrm{C}$, le poids de l'œuf répond à l'équation suivante :

Poids ouf $=59,6-1,34(0,2((T-32) \mathrm{x}$ $0,555)-16)-0,313(0,2((T-32)$ $\mathrm{x} 0,555)-16)^{2}$

Où $\mathbf{P}$ est exprimé en $\mathrm{g}$ et $\mathbf{T}$ en degré Celsius (l'équation intègre la correspondance $\left.\left[{ }^{\circ} \mathrm{C}\right]=\left(\left[{ }^{\circ} \mathrm{F}\right]-32\right) \times 5 / 9\right)$.
La diminution du poids d'œuf résulte de la réduction de l'ingéré énergétique (Balnave et Muheereza 1997) et de la synthèse protéique au fur et à mesure de l'augmentation de la température corporelle et de la mise en place des phénomènes de thermorégulation. Smith et Oliver (1972) ont dissocié l'influence de la réduction de consommation d'aliment de celle d'un effet direct des perturbations physiologiques en appariant la consommation alimentaire de poules élevées à température normale à celle de poules soumises à de fortes températures. Dans ces conditions, ils démontrent que la production d'œufs et le poids d'œuf sont influencés par la chute de consommation alimentaire contrairement à la qualité des œufs. Initialement, cette dernière dépend davantage des perturbations physiologiques. Afin de pallier cette baisse du poids d'œuf, conséquence de la réduction d'ingéré, des aliments plus concentrés (énergie et protéines) ont été distribués aux poules; cette stratégie n'atténue que partiellement la réduction du poids d'œuf (de 10 à 15\%, Joly 2003). Un enrichissement des aliments en lipides et en acide linoléique au-delà des recommandations usuelles augmente le poids des œufs de poules soumises à de fortes températures (Balnave et Brake 2005, Lin et al 2006).

\section{c) Part des compartiments de l'ouf}

Lorsque la température ambiante augmente, le poids de l'œuf mais également ceux de ses constituants sont affectés : le poids du blanc est immédiatement diminué alors que la réduction de celui du jaune apparaît progressivement 6 à 7 jours après l'introduction du stress thermique compte tenu de la durée de dépôt du vitellus (Sauveur et Picard 1987). Après quelques jours, la part du jaune semble être aussi affectée que celle de blanc (figure 6). La part relative en matière sèche du jaune et de l'albumen n'est en général pas modifiée pour des températures inférieures à $35^{\circ} \mathrm{C}$, selon ces mêmes auteurs.

Figure 6. Variation (\%) du poids des compartiments de l'œuf après introduction d'une température élevée (Sauveur et Picard 1987).

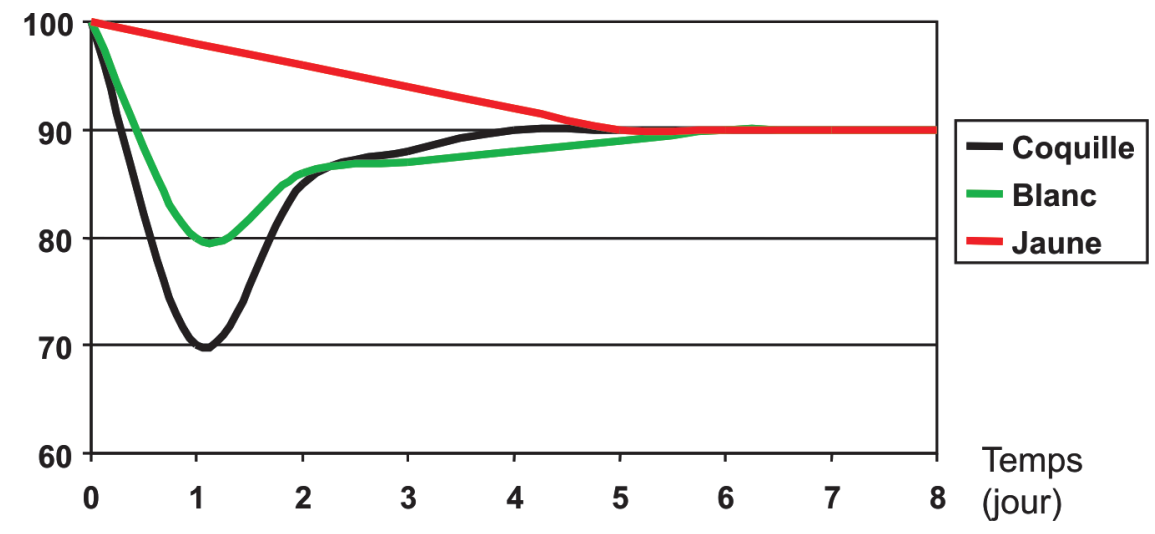


La qualité initiale de l'albumen (unité Haugh) est peu altérée par une forte température ambiante. Néanmoins, la hauteur du blanc diminue rapidement après la ponte si l'œuf reste trop longtemps stocké dans cet environnement chaud: c'est donc un effet direct de fortes températures sur l'œuf après la ponte (Sauveur et Picard 1987).

\section{d) Qualité de la coquille}

Des poules soumises à de fortes températures $\left(32-35^{\circ} \mathrm{C} / 21-24^{\circ} \mathrm{C}\right)$ réduisent de 6 à $30 \%$ ( $9 \%$ en moyenne $n=10)$ leur quantité de coquille, de 17 à $34 \%$ leur consommation alimentaire $(24,5 \%$, $n=9)$ et de 6 à $13 \%(8,4 \%, n=8)$ leur poids d'œuf (Nys 1995, réduction calculée, à partir de 10 références publiées de 1972 à 1993). La baisse de consommation d'aliment réduit la quantité de calcium ingérée et explique en partie les défauts de qualité de coquille observés lors de fortes températures d'élevage. La chaleur entraîne une diminution de l'épaisseur de coquille et, par conséquent, augmente le risque de casse. La dégradation de la qualité de coquille est moindre lorsque la chaleur est cyclique comparée à une forte chaleur constante (Balnave et Brake 2005). Cependant, une augmentation brutale de température entraîne un déclin immédiat de la qualité de coquille et ceci avant toutes modifications de quantité d'aliment ingéré. Les modifications physiologiques telles que l'alcalose respiratoire induite par le halètement ont donc un effet important sur la qualité de coquille. D'ailleurs, en cas d'ajustement alimentaire, les poules soumises à de fortes températures produisent des œufs dont la réduction de qualité est davantage marquée (Smith et Oliver 1972).

Des pratiques alimentaires permettent d'atténuer les effets néfastes de la chaleur sur la qualité de la coquille. Un apport de calcium séparé stimule la consommation d'aliment et améliore partiellement la production et le poids d'œuf (Picard et al 1993). L'apport de calcium sous forme de particules grossières de 1,5 à $3 \mathrm{~mm}$ de diamètre améliore, dans plus de $75 \%$ des très nombreux essais réalisés $(>300)$, la qualité de coquille de poules soumises à de fortes chaleurs (Guinotte et Nys 1993, Nys 1999). L'introduction d'un flash de lumière en période nocturne (Grizzle et al 1992) constitue une alternative pour favoriser la consommation d'aliment et de calcium particulaire qui contribueront à l'amélioration du poids d'œuf et de la qualité de coquille. Il n'est pas vraiment démontré qu'un apport de bicarbonate de sodium (1\%) améliore la qualité de la coquille (Balnave et Brake 2005). Un apport de $\mathrm{CO}_{2}$ dans l'eau de boisson pourrait être favorable (Odom et al 1985). Les effets bénéfiques d'apports de vitamines A et C dans l'eau de boisson sur la qualité des œufs de poules pondeuses soumises à de fortes températures sont sujets à controverses mais se révèleraient davantage convaincants pour la vitamine $\mathrm{E}$ (Lin et al 2006).

\section{2 / Les programmes lumineux}

Le contrôle de la reproduction des volailles par la photopériode a fait l'objet de nombreuses revues exhaustives de travaux principalement réalisés avant 1990. On peut citer celles de Lewis et Perry (1995), de Sauveur (1996) et de Morris (2004), à partir desquelles nous avons résumé les principaux éléments décrivant l'influence des programmes lumineux sur la qualité de l'œuf. Les efforts de sélection sur l'intensité de ponte ont réduit la dépendance saisonnière des poules mais leur sensibilité à une modification du programme lumineux reste forte puisque la synchronisation des ovipositions au sein d'une plage horaire est rapidement modifiée dès l'introduction volontaire ou accidentelle d'un changement du programme lumineux. La réglementation par l'interdiction de l'introduction de programmes fractionnés (flash nocturne) a considérablement réduit l'intérêt de leur usage.

Chez les oiseaux (Sauveur 1996, Morris 2004), la voie principale de perception de l'information lumineuse est intracrânienne; la voie oculaire, bien que secondaire, joue un rôle dans la fonction synchronisatrice des rythmes d'oviposition. En élevage, l'alternance de cycles «jour-nuit» permet de synchroniser les ovipositions des poules d'un troupeau. L'intensité lumineuse recommandée en fermes de ponte était comprise entre 10 et 15 lux maximum afin de maîtriser l'activité des animaux, leurs dépenses énergétiques sans nuire aux interventions de l'éleveur. Elle est réglementée dorénavant à 20 lux.

\section{a) Maîtrise de la maturité sexuelle}

Les programmes lumineux utilisés durant la période d'élevage des poulettes permettent de stimuler la fonction sexuelle et de mettre en place le cycle reproducteur. Une maturité sexuelle trop précoce induit, chez les oiseaux domestiques, la ponte de petits oufs, une plus grande fragilité de coquille, des troubles de l'oviposition, l'apparition de doubles ovulations ainsi qu'une augmentation de la mortalité. L'âge d'entrée en ponte des poules fait donc l'objet de contrôles stricts (Sauveur 1996). Cet effet indirect ne sera pas davantage détaillé ici.

\section{b) Photopériode et production d'œufs}

En élevage, l'effet bénéfique de la photopériode sur le taux de ponte est dû à la constance des durées d'éclairement (absence de décroissance de durée du jour). L'augmentation de la durée d'éclairement de 14 à $16 \mathrm{~h}$ voire $18 \mathrm{~h}$ n'a pas d'effet sur la ponte. Ce n'est donc pas la durée d'éclairement quotidien qui influence la reproduction mais le fait qu'il existe une phase claire 12 à $15 \mathrm{~h}$ après le réveil; l'existence de phases sombres intermédiaires n'a pas d'importance (Sauveur 1996). En cas de programmes classiques (une seule photopériode/jour), la durée de la photopériode affecte principalement la fréquence des œufs pré-fêlés in utero et des œufs déformés. L'utilisation de périodes claires de $15 \mathrm{~h}$ plutôt que $18 \mathrm{~h}$ réduit de moitié la fréquence des œufs pré-fêlés in utero. En effet, un programme de $15 \mathrm{~h}$ plonge les poules dans l'obscurité en fin de journée, donc réduit leur activité durant les premières heures de calcification, au cours desquelles la coquille en formation se révèle encore très fragile (Sauveur et Picard 1987).

Morris (2004) rapporte qu'un allongement progressif de la période claire permet un certain maintien du pic de ponte, économiquement intéressant en fin d'élevage lorsque le poids des œufs est supérieur.

\section{c) Cycles ahéméraux}

Ces cycles correspondent à une photopériode quotidienne distribuée en une seule fois mais dont la durée «jour + nuit» est légèrement inférieure ou supérieure à $24 \mathrm{~h}$ (exemple 14L:7N ou $18 \mathrm{~L}: 10 \mathrm{~N})$.

\section{$24 \mathrm{~h}$ \\ Cycles ahéméraux supérieurs à}

Les cycles de $28 \mathrm{~h}$ réduisent l'intensité de ponte et augmentent le poids d'œuf de telle sorte que la masse d'œuf exportée par la poule reste constante (Sauveur 1996). L'intensité de ponte diminue linéairement avec la longueur du cycle lorsque celle-ci augmente de 24 à $33 \mathrm{~h}$ ce qui est une résultante logique de la formation d'un œuf chaque jour et de sa durée augmentée. Le poids d'œuf s'accroît du fait de l'augmentation du temps passé dans l'oviducte : de $4-5 \%$ pour les cycles de $26 \mathrm{~h}, 6-8 \%$ pour $27 \mathrm{~h}$ et jusqu'à $11 \%$ pour ceux de $28 \mathrm{~h}$; le poids du jaune et du blanc augmentent simultanément (Sauveur et Picard 1987) alors que l'épaisseur de l'albumen (unités Haugh) diminue (Shanawany 1990). Ces longs nycthémères augmentent le poids de coquille en raison de l'accroissement du temps de séjour de l'œuf en formation 
Figure 7. Exemples de programmes d'éclairement fractionné pour poules pondeuses. Tous ces programmes s'inscrivent dans un nycthémère de 24 h ou un sousmultiple (Sauveur 1996).

\section{Fractionnement}

Témoin $14 \mathrm{~L} 10 \mathrm{~N}$

Fractionnements type 1 (exemples)

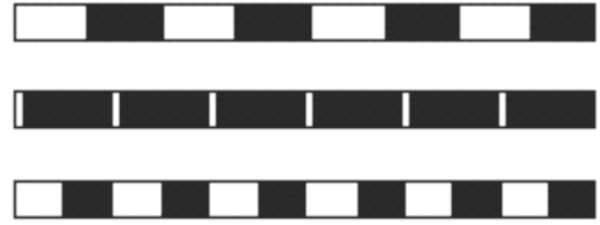

$1 / 4 \mathrm{~L} 3 \times 3 / 4 \mathrm{~N}$

$2 \mathrm{~L} 2 \mathrm{~N}$

Fractionnements type 2 (exemples)

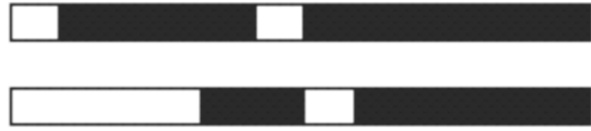

2L 8N 2L 12N

|IIIIIIIII

$8 \mathrm{~L} 4 \mathrm{~N} 2 \mathrm{~L} 10 \mathrm{~N}$

$(1 / 4 \mathrm{~L} 3 / 4 \mathrm{~N}) \times 14$

$10 \mathrm{~N}$

dans l'utérus. La réduction importante de la production d'œufs et les inconvénients pour l'éleveur du décalage des heures de soin ont prohibé l'utilisation de ses programmes lumineux qui pourraient cependant avoir un intérêt afin de corriger en fin de ponte une mauvaise qualité de la coquille. $24 \mathrm{~h}$

- Cycles ahéméraux inférieurs à

Au sein d'un troupeau, les poules qui présentent les potentialités les plus élevées (capables de faire un œuf en moins de $24 \mathrm{~h}$ ), sont pénalisées par un nycthémère normal de $24 \mathrm{~h}$ dans lequel les stimuli d'ovulation reviennent trop peu souvent. L'intérêt de raccourcir le cycle lumineux est d'obtenir un œuf toutes les

20 ou $22 \mathrm{~h}$ dans le cas où la poule très productive possède une durée de formation de l'œuf très courte. Ils ont d'ailleurs été utilisés en sélection pour détecter les poules à haute productivité. Ce cycle court améliore la coïncidence entre la période claire pendant laquelle les poules consomment l'aliment contenant le calcium et la période sombre pendant laquelle se forme la coquille. L'épaisseur de coquille est améliorée de $5 \%$ avec ce type de cycle court $(<24 \mathrm{~h})$ qui ne désynchronise pas les ovipositions du troupeau.

\section{d) Programmes fractionnés}

L'intérêt des programmes fractionnés a été exploré, il y a une trentaine d'an-

Tableau 2. Modifications des performances et de la qualité de l'œuf de poules soumises à un programme lumineux de type PLF1 pour un cycle complet 20-28 à 60-72 semaines (variation en \% des valeurs du lot témoin) (Sauveur et Picard 1987).

\begin{tabular}{|c|c|c|c|c|c|}
\hline \multirow[b]{2}{*}{ Programme } & \multirow[b]{2}{*}{$\begin{array}{c}\text { Intensité } \\
\text { de } \\
\text { ponte }\end{array}$} & \multicolumn{3}{|c|}{ Qualité de coquille } & \multirow[b]{2}{*}{$\begin{array}{l}\text { Unités } \\
\text { Haugh }\end{array}$} \\
\hline & & $\begin{array}{c}\% \text { de } \\
\text { coquille } \\
\text { densité } \\
\text { ou index }\end{array}$ & $\begin{array}{l}\text { Poids de } \\
\text { coquille ou } \\
\text { épaisseur }\end{array}$ & $\begin{array}{l}\text { Résistance } \\
\text { rupture }\end{array}$ & \\
\hline 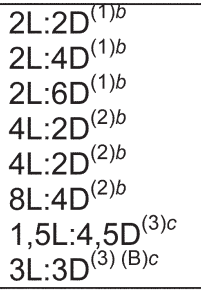 & $\begin{array}{l}-6,7 \\
-4,1 \\
-2,0 \\
-11,0 \\
-11,0^{*} \\
-6,8^{\star} \\
-6,5^{\star} \\
-5,5^{\star}\end{array}$ & $\begin{array}{l}+5,6^{*} \\
+3,0^{*} \\
+4,3^{*} \\
+6,7^{*} \\
+4,3^{*}\end{array}$ & $\begin{array}{l}+6,0^{*} \\
+5,4^{*} \\
+4,2^{*} \\
+9,7^{*} \\
+7,5^{*} \\
+7,5^{*} \\
+9,6^{*} \\
+7,7^{*}\end{array}$ & $\begin{array}{l}+7,8^{*} \\
+1,1 \\
+3,7^{*} \\
+13,6^{*} \\
+11,0^{*}\end{array}$ & $\begin{array}{c}-2,5^{*} \\
-1,9^{*} \\
-1,7^{*} \\
-1,5 \\
0 \\
-1,5\end{array}$ \\
\hline
\end{tabular}

* Variation significative par rapport au lot témoin.

b OEufs blancs ; c œufs colorés.

(B) : Evoluant ensuite jusqu'à 1,5L : 4,5D.

(1) : Cooper et Barnet 1977, (2): Torges, Rauch et Wegner 1981, (3) : Sauveur et Mongin 1983. nées, essentiellement comme moyen de contrôle de la surconsommation alimentaire des poules pondeuses. Leur utilisation est limitée en Europe par la législation bien-être qui interdit l'introduction de flash lumineux pendant la nuit. Deux types de Programmes Lumineux Fractionnés (PLF) sont à distinguer selon Sauveur (1996, figure 7).

Les Programmes Lumineux Fractionnés de type I (PLF I) dits «symétriques» sont constitués de séquences claires et obscures de courtes durées qui se répètent régulièrement à l'intérieur d'une période de $24 \mathrm{~h}$. Il n'existe pas de nuit principale et les ovipositions des poules du troupeau sont désynchronisées sur 24 h (tableau 2).

La désynchronisation de la ponte induite par les PLF1 entraîne une légère diminution de l'intensité de ponte (2 à 4\% s'ils sont appliqués en début de période de reproduction) car la durée de formation de l'œuf augmente comme si les poules étaient placées en lumière permanente. Par conséquent, le poids d'œuf augmente en lien avec l'augmentation du poids des trois compartiments de l'œuf: jaune, blanc et coquille. Ces programmes favorisent une ingestion du calcium pendant la formation de la coquille, qui coïncide avec la nuit en programme lumineux classique.

Les PLF1 se révèlent faciles à mettre en place en bâtiment obscur et ne désynchronisent pas le travail de l'éleveur. La réponse des poules à ces programmes dépend cependant de la qualité de l'isolation phonique et lumineuse du bâtiment. D'un point de vue pratique, il est intéressant de noter qu'il est possible d'introduire un programme lumineux fractionné pour une durée limitée afin de corriger un problème de qualité d'œufs (poids ou coquille) sans que cela ne perturbe notablement la production d'œufs.

Les Programmes Lumineux Fractionnés de type II ( $P L F$ 2) dits «dissymétriques» sont caractérisés par l'insertion d'une succession de courtes périodes obscures lors de la phase claire. Une période de nuit principale subsiste $(8 \mathrm{ou} 10 \mathrm{~h})$ permettant la synchronisation des ovipositions comme dans un programme classique. Ces programmes ne modifient ni l'intensité de ponte, ni le poids de l'œuf ou la solidité de coquille. Ils ont été développés pour rationner les poules.

Le plus connu est le programme dit «biomittent» coupant chacune des $16 \mathrm{~h}$ claires d'un nycthémère normal en $15 \mathrm{~min}$ de lumière et $45 \mathrm{~min}$ d'obscurité. Comparé au PLFI, ce programme induit une légère baisse de poids d'œuf 
Tableau 3. Performance et qualité de l'œuf de poules soumises à 3 programmes lumineux fractionnés de 23 à 72 semaines d'âge (Morris 2004).

\begin{tabular}{|c|c|c|c|}
\hline Programmes lumineux & $\begin{array}{c}\text { Biomittent } \\
\text { dissymétrique }\end{array}$ & $\begin{array}{c}4(3 \mathrm{~L}: 3 \mathrm{~N}) \\
\text { Symétrique }\end{array}$ & $\begin{array}{c}24(0,25 \mathrm{~L}: 0,75 \mathrm{~N}) \\
\text { Symétrique }\end{array}$ \\
\hline $\begin{array}{l}\text { Taux de ponte }(\%) \\
\text { Poids moyen des œufs (g) } \\
\text { Masse d'œuf exportée } \\
\text { (g/poule/j) } \\
\text { Consommation d'aliment } \\
\text { (g/poule/j) } \\
\text { Epaisseur de coquille } \\
\text { à } 65 \text { semaines d'âge } \\
\text { des poules }\left(\mathrm{mg} / \mathrm{cm}^{2}\right)\end{array}$ & $\begin{array}{r}73,9 \\
62,1 \\
49,5 \\
109 \\
79,3 \\
\end{array}$ & $\begin{array}{l}72,1^{*} \\
63,6^{*} \\
49,4 \\
112^{*} \\
82,3^{*}\end{array}$ & $\begin{array}{l}72,3^{*} \\
63,5^{*} \\
49,5 \\
108 \\
81,5^{*}\end{array}$ \\
\hline
\end{tabular}

* : Significativement différent du programme «Biomittent».

et de l'épaisseur de coquille, associée à une augmentation du taux de ponte. La masse d'œuf exportée reste, cependant, inchangée (Morris 2004, tableau 3).

Ces programmes d'éclairement fractionnés tendent à accroître la viabilité des poules, à diminuer la fréquence des tics et des poux. Ils limitent aussi les répercussions physiologiques des chocs thermiques. Néanmoins, la directive 1999/74/CE, imposant une nuit ininterrompue voisine de $8 \mathrm{~h}$, interdit l'usage des programmes fractionnés symétriques en élevage malgré leur intérêt pour la santé des animaux. Quant aux programmes fractionnés dissymétriques, ils ont perdu de leur intérêt car le rationnement des poules n'est plus vraiment d'actualité, suite à la sélection de poules sur une faible consommation alimentaire pour réduire leur indice de consommation.

\section{3 / Le système d'élevage}

Afin de favoriser le développement d'élevage respectueux du bien-être des poules, la Commission Européenne (Directive 1999/74/CE) impose l'abandon de l'élevage en cages conventionnelles, à échéance de 2012, pour des systèmes de cages aménagées ou volière, avec ou sans parcours (Guémené 2007). Selon une collecte de données européennes et françaises, $32 \%$ des pondeuses étaient élevées en systèmes alternatifs au sein de l'UE à 27 en 2008 (Magdelaine et Braine 2010). L'Allemagne est le principal producteur d'œufs alternatifs au sein de l'UE, devant les Pays-Bas, le Royaume-Uni et la France. La proportion de pondeuses en modes d'élevage alternatifs se stabilise autour de $20 \%$ en France. Les effectifs estimés en 2008 étaient de 1,7 millions de pondeuses bio, 1,6 millions de pondeuses Label Rouge, 3,6 millions de pondeuses plein air (hors LR) et 1,3 millions de pondeuses au sol (ITAVI 2009).
Dans ce chapitre, nous étudierons la qualité des œufs issus de cages conventionnelles par rapport à celle de poules ayant un accès à un parcours ou élevées en cages aménagées. Leur impact sur la qualité hygiénique de l'œuf est décrit dans un autre article de ce numéro spécial (Mallet et al 2010).

a) Comparaison d'œufs de poules élevées en cages ou au sol, avec ou sans parcours.

Classiquement, un élevage en cages augmente le poids de l'œuf de 1 à $2 \%$, mais Sauveur (1991) souligne la variabilité de cet effet et la présence de réductions importantes pouvant attendre $10 \%$ dans certains élevages fermiers, du fait des pratiques d'élevage. Cette réduction quand elle est présente, est associée à une diminution de la part de jaune. En revanche, aucune variation répétable de la composition de l'œuf, teneurs en lipides ou en protéines ou encore en matière sèche n'est mise en évidence (Sauveur 1991, Hidalgo et al 2008), en accord avec la stabilité des constituants majeurs de l'œuf (Nys et Sauveur 2004). Pour les acides gras de l'œuf et les constituants mineurs dont la teneur dans l'œuf est directement influencée par l'alimentation de la poule, les variations révélées par les publications comparant la composition d'œufs issus des différents systèmes d'élevage, se caractérisent par leur inconstance et l'absence d'une tendance en faveur d'un système (Sauveur 1991, Rossi 2007). La composition globale de l'œuf est en effet indépendante du type de logement des poules dans la mesure où l'alimentation est identique. Cependant, l'accès au parcours donne la possibilité aux poules de consommer des matrices environnementales (sol, herbe, vers, insectes) et pourrait, de ce fait, favoriser l'ingestion de substances indésirables, telles que les polluants organiques persistants (Jondreville et al 2010). De même, les variations de qua- lité de l'albumen semblent imprévisibles entre modes d'élevage et apparaissent liées au temps passé par les poules sur leurs œufs à l'intérieur du nid. La coloration du jaune, directement liée à l'alimentation, se révèle plus intense lorsque les poules ont accès à un parcours, en comparaison d'œufs produits en cages si les deux lots expérimentaux reçoivent le même aliment de base. En revanche, les sondages sur des œufs de consommation révèlent une couleur $\mathrm{du}$ jaune plus intense en élevage conventionnel suite à une moindre supplémentation en pigments rouges dans les élevages bio ou label du fait de règlement interne à ces productions. La coloration du jaune reflète donc la quantité de caraténoïdes ingérée, qu'ils proviennent de l'herbe consommée ou de la supplémentation en xanthophylles des aliments pondeuses. Les données de coloration de coquille et d'inclusions dans les œufs ne sont pas interprétables car trop variables d'une étude à l'autre (Sauveur 1991). Hidalgo et al (2008) montrent également que le mode d'élevage a peu d'influence sur la qualité technologique de l'œuf.

La solidité de la coquille ne varie pas de façon constante entre études. Néanmoins, l'augmentation du nombre d'œufs cassés en fin de ponte est atténuée dans des élevages en plein air ou sur litière selon certains auteurs cités par Sauveur (1991). Selon cet auteur, l'augmentation limitée mais significative de la résistance à la rupture de la coquille, en élevage plein air résulterait d'une température ambiante inférieure dans les élevages plein air et d'une consommation d'aliment plus élevée. Cet avantage n'a pas été confirmé dans des travaux plus récents (EFSA 2005, Michel et al 2007). Par ailleurs, en volière, le pourcentage d'œufs pondus au sol et souillés augmente le taux de déclassement des œufs d'environ 2\% avec de grandes variations entre élevages (Fiks-Van Niekert 2005).

En conclusion, il est donc difficile de comparer les résultats entre ces diverses études du fait de la diversité des origines génétiques, de l'âge et surtout de l'alimentation des poules (aliment distribué par l'homme ou à disposition sur le parcours : sol, herbe, pédofaune...). L'opinion favorable dont jouit l'œuf «plein air» dans l'esprit des consommateurs ne s'appuie donc pas sur des différences nutritionnelles ou qualitatives objectives mais plus sur une perception très positive du système d'élevage. De plus, il est à noter qu'au sein d'un système de production donné, l'effet éleveur sur la qualité de l'œuf peut être davantage marqué que celui du système de production lui-même (Rossi 2007). 
b) Comparaison d'œufs de poules élevées en cages conventionnelles ou en cages aménagées

Les cages conventionnelles des poules pondeuses sont substituées en partie par des cages aménagées, c'est-à-dire équipées de perchoirs, d'un nid et d'une surface de grattage et de picotage. Les performances zootechniques et la qualité de l'œuf ont été étudiées dans des réseaux d'élevages équipés de cages aménagées en France (Mirabito et al 2005, 2007, Michel et al 2007, Guémené 2007), en Suède (Tauson et al 2002), en Allemagne (Rauch et al 2002) et au Royaume-Uni (Appleby et al 2002).

Ces auteurs n'ont pas observé de modifications de la productivité des poules ou de leur mortalité. Cependant, cette dernière est plus imprévisible qu'en élevage conventionnel du fait notamment du risque de picage (Michel et al 2007, Guémené 2007). Ces travaux indiquent que progressivement, les aménagements des systèmes de cages aménagées s'améliorent et conduisent comme dans l'exemple suédois, aux performances standards de la souche. De même, Mirabito et al ont observé lors de la première campagne une réduction du poids d'œuf d'environ $1,2 \%$ en fin de ponte en cages aménagées, qu'ils n'ont pas confirmée lors de la seconde campagne (Mirabito et al 2005, 2007).

La plupart des études initiales sur les cages aménagées révèlent également une plus grande fréquence d'œufs cassés/fêlés (4 à $34 \%$ ) ou sales (7 à 26\%) par rapport aux cages conventionnelles.
Ce déclassement est particulièrement important pour les œufs pondus hors du nid et dépend de l'hygiène des cages (Rauch et al 2002, Tauson et al 2002, Mirabito et al 2005, 2007, Michel et al 2007). Il est à noter une grande variabilité entre les différents modèles de cages aménagées. En effet, le type de nid, son positionnement dans la cage, l'intensité lumineuse à l'intérieur du nid, le type de fond du nid ont un effet sur son attractivité et par conséquent son utilisation. $A$ contrario, lorsque la ponte au nid est élevée, l'accumulation d'œufs devant le nid et l'absence d'avancement automatique des bandes à œufs sont des facteurs d'explication de la présence d'un plus grand nombre d'œufs fêlés voire cassés par picage (Mirabito et al 2005).

Il apparaît donc que les dispositifs de cages aménagées peuvent influencer la qualité des œufs (fréquences d'œufs sales et cassés) selon la conception de la cage, la disposition des aménagements et la taille du groupe, la présence d'un fil ralentisseur de roulement des œufs ou le rythme d'avancement de la bande collectant les œufs. Des aménagements issus de ces études initiales ont été, pour la plupart, intégrées par les fabricants de cages. Ceux-ci optimisent progressivement les combinaisons d'aménagement pour obtenir les meilleures performances. Néanmoins, diverses options restent discutées telles que les conditions d'élevage des poulettes, le choix de la taille de groupe optimale pour l'hygiène de la cage, la gestion du risque de picage. De nouvelles stratégies sont également testées afin d'intégrer la surface de grattage picotage et/ou d'optimiser l'utilisation du nid (cage dissymétrique, Bignon et al 2009) afin de proposer les systèmes les plus favorables à la production et à la qualité de l'œuf.

\section{Conclusion}

L'animal lui-même, au travers de modifications physiologiques, du fait d'interactions génotype $\times$ milieu ou de réactions à son environnement direct, influe de manière significative sur la qualité de l'œuf. En élevage, les influences de l'âge sur l'œuf sont parfaitement connues mais davantage subies que maîtrisées. Les éleveurs peuvent induire une mue sur leur troupeau afin de prolonger la production des poules et de répondre à la demande des consommateurs de gros calibre tout en maîtrisant le taux d'œufs déclassés. L'impact des facteurs environnementaux a été analysé depuis de nombreuses années et des mesures d'élevage permettent de les contrôler pour maintenir la qualité de l'œuf. Les éleveurs sont bien conscients qu'une erreur de management (programme lumineux, température) peut entraîner des pertes économiques non négligeables à l'échelle de l'élevage. Le défi des éleveurs est, aujourd'hui, de prendre en compte la réglementation pour le bien-être des poules et par conséquent, de s'adapter à de nouveaux systèmes de production. De plus, il est essentiel de trouver de nouvelles méthodes pour pallier l'abandon de pratiques assurant un contrôle de la qualité de l'œuf comme cela a été le cas pour les programmes lumineux fractionnés.

\section{Normes $550 \mathrm{~cm}^{2}$}

Code 3

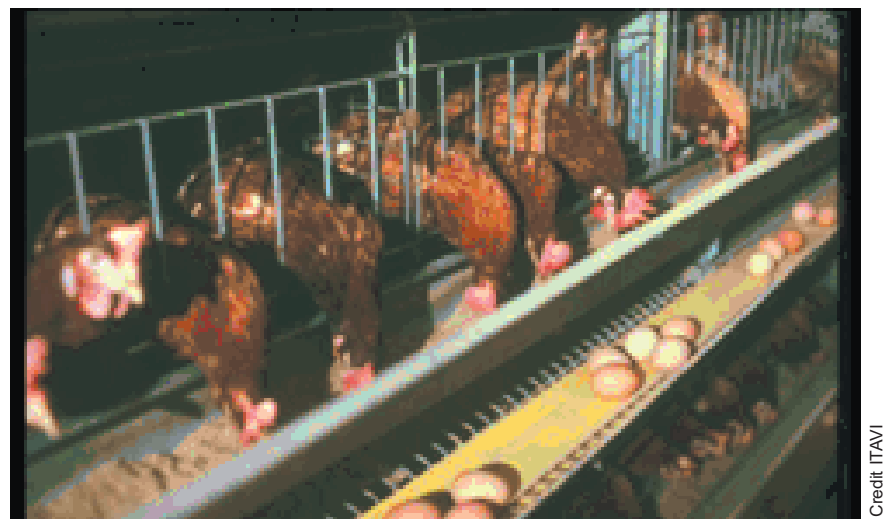

Normes 2012

Code 3

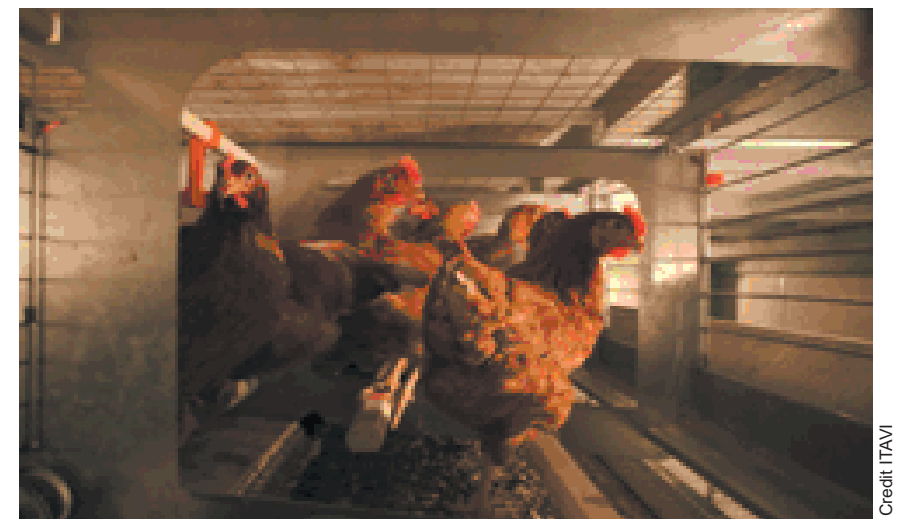




\section{Références}

ADAS, 1989. Food, farming land and leisure, Nottingham GB: Albumen Quality Chart.

Ahmed A.M., Rodriguez-Navarro A.B., Vidal M.L., Gautron J., Garcia-Ruiz J.M., Nys Y., 2005. Changes in eggshell mechanical properties, crystallographic texture and in matrix proteins induced by moult in hens. Brit. Poult. Sci., 46, 268-279.

Appleby M.C., Walker A.W., Nicol C.J., Lindberg A.C., Freire R., Hughes B.O., Elson H.A., 2002. Development of furnished cages for laying hens. Brit. Poult. Sci., 43, 489-500.

Balnave D., Brake J., 2005. Nutrition and management of heat-stressed pullets and laying hens. World's Poult. Sci., 61, 399-406.

Balnave D., Muheereza S.K., 1997. Improving eggshell quality at high temperatures with dietary sodium bicarbonate. Poult. Sci., 76, 588-593.

Beaumont C., Calenge F., Chapuis H., Fablet J., Minvielle F., Tixier-Boichard M., 2010. Génétique de la qualité de l'œuf. In : Numéro Spécial, Qualité de l'oeuf. Nys Y. (Ed). Inra Prod. Anim., 23, 123-132.

Bell D.D., 2003. Historical and current molting practices in the u.s. table egg industry. Poult. Sci., 82, 965-970.

Bignon L., Guémené D., Couty M., Mirabito L., 2009. La cage idéale pour poules pondeuses: Mythe ou réalité ? gèmes Journ. Rech. Avicole, Saint-Malo, France, 2009/03/25-26, 32 (Résumé). CDRom : 000098.pdf, 80-84.

Cooper J.B., Barnett B.D., 1977. Photoperiod study with chicken hens. Poult. Sci., 56, 1832-1835.

Curtis P.A., Kerth L.K., Anderson K.E., 2005. Quality and compositional characteristics of laver hens as affected by bird age P.A. Proc. $11^{\text {th }}$ Eur. Symp. Quality of Eggs and Egg Products, Doowerth, The Netherland, 214-219.

EFSA (European Food Safety Authority), 2005. The welfare aspects of various systems for keeping laying hens. Scientific report, EFSA-Q-2003-92, 143p.

Fiks-Van Niekerk T.H.G.C.M., 2005. Housing systems for laying hens and their effect on egg quality. Proc. $11^{\text {th }}$ Eur. Symp. Quality of Eggs and Egg Products, Doowerth, The Netherland, 262-266.

Garlich J., Brake J., Parkhurst C.R., Thaxton J.P., Morgan G.W., 1984. Physiological profile of caged layers during one production year, molt and postmolt: egg production, egg shell quality, liver, femur, and blood parameters. Poult. Sci., 63, 339-343.

Goto K., Harris G.C., Waldroup P.W., 1979. Relationship between pimpling of egg shells, environmental temperature and carbonic anhydrase activity of certain body tissues. Poult. Sci., 58, 1014 .

Grizzle J., Theanacho M., Saxton A., Broaen J., 1992. Nutritional and environmental factors involved in egg shell quality of laying hens. Brit. Poult. Sci., 33, 781-794.

Guémené D., 2007. Situation actuelle et évolution des systèmes d'élevage pour poules pondeuses en Europe. TeMA, 4, 4-10.

Guinotte F., Nys Y., 1993. Factors affecting the intestinal calcium availability in laying hens: consequences on shell quality. Proc. $V^{\text {th }}$ Eur. Symp. on the Quality of egg and eggs products, Nys Y. (Ed), Tours, France, 149-162.

Hidalgo A., Rossi M., Clerici F., Ratti S., 2008. A market study on the quality characteristics of eggs from different housing systems. Food Chem., 106, 1031-1038.

ITAVI, 2009. Situation de la production et des marchés des œufs et des produits d'œufs. Note réalisée par le service économie de I'ITAVI en octobre 2009, 25p.

Joly P., 2003. Conduite technique des élevages de poulette et de pondeuses en clima chaud. In : La Production d'œufs de Consommation en Climat Chaud. Amand C., Aubert C., Braine A., Cardinale E. Champagne J., Comte S., Dayon J.F., Drouin J.P., Goater E., Guerder F., Guibert F.M., Joly Y., Magdelaine P., Renault P., Valancony H., Fleury V. (Eds), Paris, France, 53-61.

Jondreville C., Fournier A., Travel A., Feidt C., Roudaut B., 2010. Modalités et risques de transfert des contaminants chimiques vers l'œuf de poule pondeuse. In : Numéro Spécial, Qualité de l'oeuf. Nys Y. (Ed). Inra Prod. Anim., 23, 205-214.

Journal Officiel des Communautés Européennes (1999). Directive 99/74/CE du Conseil du 19-07-99 concernant les volailles de l'espèce Gallus gallus élevées pour la ponte. JO L 203/53 du 03/08/99, 53-57.

Kerth L.K., Curtis P.A., Anderson K.E., 2005. Impact of bird age on the functionality of eggs from current layer strains. Proc. 11 th Eur. Symp. Quality of Eggs and Egg Products, Doowerth, The Netherland, 32-36.

Kuenzel W.J., Wideman R.F., Chapman M. Golden C., Hooge D.M., 2005. A practical method for induced moulting of caged layers that combines full access to feed and water, dietary thyroactive protein, and short day length. World's Poult. Sci. J., 61, 599-624.

Landers K. L., Howard Z.R., Woodward C.L., Birkhold S.G., Ricke S.C., 2005. Potential of alfalfa as an alternative molt induction diet for laying hens: egg quality and consumer acceptability. Bioresource Technol., 96, 907-911.

Lewis P.D., Perry G.C., 1995. Effects of lighting on reproduction in poultry. In: Poultry Production, Hunton P. (Ed). Elsevier, Amsterdam, World Anim. Sci., 9, 359-388.

Lin H., Jiao H.C., Buyse J., Decuypere E., 2006. Strategies for preventing heat stress in poultry. World's Poult. Sci., 62, 71-85.

Magdelaine P., Braine A., 2010. Production et consommation des œufs et des ovoproduits. In : Numéro Spécial, Qualité de l'oeuf. Nys Y. (Ed). Inra Prod. Anim., 23, 111-122.

Mallet S., Huneau-Salaün A., De Reu K., 2010. Système d'élevage et qualité hygiénique de l'œuf. In: Numéro Spécial, Qualité de l'oeuf. Nys Y. (Ed). Inra Prod. Anim., 23, 183192.

Mansoori B., Modirsanei M., Farkhoy M., Kiaei M.M., Honazard J., 2007. The influence of different single dietary sources on moult induction in laying hens. J. Sci. Food Agric., 87, 2555-2559.

Mardsen A., Morris T.R., 1987. Quantitative review of the effect of environmental tempera- ture on food intake, egg output and energy balance in laying pullets, Brit. Poult. Sci., 28, 693-704.

Mertens K, Bain M., Perianu C., De Baerdemaeker J., Decuypere E., De Ketelaere B., 2010. Qualité physico-chimique de l'œuf de consommation. In : Science et technologie de l'œuf et des ovoproduits. Nau F., GuérinDubiard C., Baron F., Thapon J.L. (Eds). Tec et Doc Lavoisier, Paris, France, 1, Chap. 7, 265314.

Michel V., Arnould C., Mirabito L., Guémené D., 2007. Système de production et bien-être en élevage de poules pondeuses. INRA Prod. Anim., 20, 47-52.

Mills A.D., Nys Y., Gautron J., Zawadski J., 1991. Whitening of brown shelled eggs: individual variation and relationships with age, fearfulness, oviposition interval and stress. Brit. Poult. Sci., 32, 117-129.

Mirabito L., Coignard S., Travel A., 2005. Effet du mode de logement des poules pondeuses d'œufs de consommation (cages aménagées $v s$ cages conventionnelles) sur les performances zootechniques et divers critères de qualité des oufs. Résultats d'une étude en élevage de production. 6èmes Journ. Rech. Avicole, Saint-Malo, France, 56-61.

Mirabito L., Giraud S., Travel A., 2007. Effet du mode de logement des poules pondeuses d'œufs de consommation (cages aménagées $v s$ cages conventionnelles) sur le comportement des poules et divers critères de qualité des œufs, TeMa, 2, 10-18.

Mongin P., 1978. Acid-base balance during egoshell formation. In: Respiratory function of birds, Adult and Embryonic. Piiper J. (Ed). Springer Verlag, Berlin, 247-259.

Morris T.R., 2004. Environmental control for layers. World's Poult. Sci. J., 60, 163-175.

Nys Y., 1986. Relationships between age, shell quality and individual rate and duration of shell formation in domestic hens. Brit. Poult. Sci., 27, 253-259.

Nys Y., 1995. Influence of nutritional factors on eggshell quality at high environmental temperature. $6^{\text {th }}$ Symp.Quality of Eggs and Egg Products, Zaragoza, Espagne, 25-29 septembre, Cepero Briz R. (Ed). WPSA, Spanish Branch, 209-220.

Nys Y., 1999. Nutritional factors affecting eggshell quality. Czech J. Anim. Sci., 44, 135 143.

Nys Y., 2010. Structure et formation de l'œuf. In : Science et technologie de l'œuf et des ovoproduits. Nau F., Guérin-Dubiard C., Baron F., Thapon J.L. (Eds). Tec. et Doc Lavoisier, Paris, France, 1, Chap. 5, 141-152.

Nys Y, Sauveur B., 2004. Valeur nutritionnelle des œufs. INRA Prod Anim, 17, 385-393.

Nys Y., Hincke M.T., Arias J.L., Garcia-Ruiz J.M., Solomon S.E., 1999. Avian eggshell mineralization. Avian Poult. Biol. Rev., 10, 143166.

Nys Y., Burlot T., Dunn I.C., 2008. Internal quality of eggs: any better, any worse? $23^{\text {th }}$ World's Poultry Congress. 30 june-7 july, Brisbane, Australie, Australian branch, (CDRom (papers $\|$ wpc08Final00034), 10p.

Odom T.W., Harrison P.C., Darre M.J., 1985. The effect of drinking carbonated water on the 
egg shell quality of single comb white leghorns hens exposed to high environmental temperature. Poult. Sci., 64, 594-596.

Picard M., Sauveur B., Fenardji F., Angulo I., Mongin P., 1993. Ajustements technico-économiques possibles de l'alimentation des volailles dans les pays chauds. INRA Prod. Anim., 6, 87-103.

Rauch H.W., Buchenauer D. Hartung J., Hinrichs P., 2002. Experiences from various furnished cage models, $11^{\text {th }}$ Eur. Poult. Conf., Bremen, Germany, Cd-Rom.

Roberts J.R., Brackpool C.E., 1994. The ultrastructure of avian egg shells. Poult. Sci. Rev., 5, 245-272.

Rodriguez-Navarro A., Kalin O., Nys Y., Garcia-Ruiz J.M., 2002. Influence of the microstructure on the shell strength of eggs laid by hens of different ages. Brit. Poult. Sci., 43, 395-403.

Romanoff A.L, Romanoff A.J., 1949. The Avian Egg. John Wiley \& Sons, NY, USA, $998 \mathrm{p}$

Rossi M., 2007. Influence of the laying hen systems on table egg characteristic. Proc. $18^{\text {th }}$ Eur. Symp. Quality of Poultry Meat and $12^{\text {th }}$ Eur. Symp. Quality of eggs and Egg products, Prague, September 2-5, 49-51.

Sauveur B., 1988. Reproduction and egg production in poultry. Reproduction des volailles et production d'œufs, INRA Editions, Paris, France, 449p.
Sauveur B., 1991. Mode d'élevage des poules et qualité de l'œuf de consommation. INRA Prod. Anim., 4, 123-130.

Sauveur B., 1996. Photopériodisme et reproduction des oiseaux domestiques femelles. INRA Prod. Anim., 9, 25-34.

Sauveur B., Mongin P., 1983. Performance of layers reared and/or kept under different 6 hour light-dark cycles. Brit. Poult. Sci., 24 405-416.

Sauveur B., Picard M., 1987. Environnental effects on egg quality. In: Egg quality curren problems and recent advances. Well R.G., Belyavin C.G. (Eds). chap. 14, 219-234

Scheideler S.E., Jaroni D., Froning G., 1998 Strain and age effects on egg composition from hens fed diets rich in n-3 fatty acid. Poult. Sci., 77, 192-196.

Shanawany M.M., 1990. Ahemeral light cycles and egg quality. World's Poult. Sci. J., 46, 101-108.

SNIPO (Syndicat National des Industriels et Professionnels de l'œuf, F. Fégor), 2008. Note 08001 : Matière sèche des ovoproduits et appellation légale. Commission Technique ovoproduits, 15 octobre, $1 \mathrm{p}$.

Smith A.J., Oliver J., 1972. Some nutritional problem associated with egg production at high environmental temperatures. 4. The effect of prolonged exposure to high environmental temperatures on the productivity of pullets fed on high energy diets. Rhod. J. Agric. Res., 10, $43-60$
Solomon S.E., 1991. Egg and eggshell quality. Wolfe publishing LTD, London, England, 149 p.

Tauson R., Holm K.E., Wall H., 2002. Experiences from various furnished cage models in Sweden. 11 th Eur. Poult. Conf., Bremen, Germany, Cd-Rom.

Ternes W., Acker L., Scholtyssek S., 1994. Ei und Eiprodukte. Verlag Paul Parey, 494p.

Torges H.G., Rauch H.W., Wegner R.M.,1981. Intermittieren de Beleuchtung von Legehennen und ihr Einfluss auf Legeleistung, Eiqualität, Eiablage, und Futteraufnahmerhythmik. Arch. Geflügelk., 45, 76-82.

Travel A., Bedos M., Narcy A., Lescoat P., Schlegel P., Durosoy S., Nys Y., 2009. Influence of selenium source and level on laying hens performances, egg and tissue se contents and oxidative status under high ambient temperature. 17 $7^{\text {th }}$ Eur. Symp. Poult. Nutr., Edinburgh, Scotland, 250.

Wolfenson D., Frei Y.F., Snapir N., Berman A., 1981. Heat stress effects on capillary blood flow and its redistribution in the laying hen. Pflugers Arch., 390, 86-93.

Yahav S., 2009. Alleviating heat stress in domestic fowl: different strategies. World's Poult. Sci. J., 65, 719-732

\section{Résumé}

La qualité de l'œuf se révèle fortement influencée par les modifications physiologiques liées à l'âge de la poule, à une mue ou par les conditions environnementales (température, lumière et système d'élevage). Le poids de l'œuf augmente avec l'âge des poules, du fait notamment d'un accroissement de la part de jaune. L'âge de la poule réduit la qualité de la coquille ainsi que les propriétés fonctionnelles des œufs. La mue restaure, pour un cycle plus court, les performances des poules et la qualité des oeufs, dégradée en fin de premier cycle. L'augmentation de la température ambiante en élevage entraîne, dès $30^{\circ} \mathrm{C}$, des perturbations de l'équilibre acido-basique et une chute de la consommation d'aliment. Celles-ci réduisent la production d'œufs, leur poids et leur qualité de coquille. Les programmes lumineux mis en place avant et après l'entrée en ponte, conditionnent la courbe de ponte. L'application de nycthémères supérieurs à $24 \mathrm{~h}$ ou de programmes fractionnés symétriques augmente le poids d'œuf et l'épaisseur de coquille mais les directives européennes interdisent leur usage. La qualité sensorielle, nutritionnelle ou technologique de l'œuf est faiblement affectée et de manière inconstante par le système de production, volière ou cage. Certaines cages aménagées ont initialement engendré une détérioration de l'intégrité des oufs mais des modifications de leur conception, de la disposition des aménagements et de la taille du groupe aboutissent progressivement à l'obtention de résultats comparables à ceux obtenus en cages conventionnelles.

\section{Abstract}

\section{Physiological and environmental factors affecting egg quality}

Egg quality is greatly influenced by hen physiology, including age, moult and by environmental conditions (temperature, lighting cycle and rearing system). Egg weight increases with hen age, partly due to the increased yolk proportion. Eggshell quality and functional properties of eggs are progressively impaired with hen age. Moult restores, for a shorter production cycle, the progressive degradation in egg production and quality observed at the end of the laying period. High ambient temperature $\left(>30^{\circ} \mathrm{C}\right)$ elicits in hens changes in acido-basic balance and in feed consumption. These changes reduce egg production, egg weight and eggshell strength. Lighting programs applied during the rearing and production periods of hens influence egg production. Ahemeral (>24 h) and symmetrical cycle programs increase egg weight and eggshell thickness but recent EU directives banned their use. The production system has limited and inconsistent effects on sensorial, nutritional or functional properties of eggs. Furnished cages initially resulted in higher percentages of downgraded eggs but progressive improvements in cage design, of equipments distribution and changes in hen group size allow similar performance to conventional cages if hens are beak trimmed.

TRAVEL A., NYS Y., LOPES E., 2010. Facteurs physiologiques et environnementaux influençant la production et la qualité de l'œuf. In : Numéro Spécial, Qualité de l'œuf. Nys Y. (Ed). Inra Prod. Anim., 23, 155-166. 